\title{
Análise da taxa de variação da linha de costa da Enseada de Tijucas (SC) em diferentes escalas temporais como indicadora de suscetibilidade costeira Analysis of the Enseada de Tijucas (SC) shoreline change rate in different time scales as indicator of coastal susceptibility
}

Elaine Cristina Santos ${ }^{\text {ab }}$, Jarbas Bonetti ${ }^{\text {ac }}$

aLaboratório de Oceanografia Costeira - OCN/CFM, Universidade Federal de Santa Catarina

belaine-cristinas@hotmail.com,cjarbas.bonetti@ufsc.br

\begin{abstract}
Resumo
A Enseada de Tijucas, localizada no interior da baía homônima, pode ser considerada um ambiente costeiro semiabrigado em relação à incidência direta de ondas. Entretanto, encontra-se suscetível a eventos de inundação costeira associados à ocorrência de maré meteorológica. Dentre os descritores que podem ser avaliados para melhor identificar áreas suscetíveis a estes eventos destaca-se a análise da taxa de variação da posição da linha de costa através de aerofotografias/imagens de satélite. A rotina Digital Shoreline Analysis System possibilita esta análise a partir da vetorização das linhas de costa de datas distintas e foi escolhida para auxiliar na investigação sobre os eventos de inundação costeira na área de estudo. Foram utilizadas aerofotografias de 1938, 1957, 1978 e 2010, e as imagens de satélite de 2005, 2009, 2011, 2014 e 2015, tendo-se efetuado processamentos independentes para estas duas séries. A análise das aerofotografias resultou na identificação de um processo de avanço da linha de costa, com taxa média de $+1,62 \mathrm{~m} /$ ano. Nas imagens de satélite as variações na posição da linha de costa foram menores, com taxa média de $+0,04 \mathrm{~m} / \mathrm{ano}$. A realização das duas análises em escalas temporais distintas permitiu identificar padrões de deslocamento diferenciados para uma mesma linha de costa. As modificações foram expressivas na escala interdecadal coberta pelas aerofotografias, sugerindo a existência de uma tendência de avanço da linha de costa de longo prazo. Nos 10 anos de análise das imagens de satélite as modificações foram mais sutis, com variações irregulares, ressaltando padrões de alta frequência possivelmente associados a eventos específicos.
\end{abstract}

Palavras-chave: inundação costeira; DSAS; análise espacial.

\begin{abstract}
Enseada de Tijucas, located in the innermost part of the homonymous bay, can be considered a semisheltered coastal sector regarding the direct wave incidence. However, it is susceptible to events such as coastal inundation associated with storm surges. Among the descriptors that could be used to identify susceptible areas to these events, shoreline change rates analysis from aerial photographs/satellite images can be underlined. The Digital Shoreline Analysis System GIS extension performs this analysis from the shoreline vectorization. This technique was chosen to support coastal inundation patterns investigation. Aerial photographs from 1938, 1957, 1978 and 2010 were used, as well as satellite images from 2005, 2009, 2011, 2014 and 2015, which were independently processed. Aerial photographs analysis allowed positive shoreline displacement tendency identification, with $+1.62 \mathrm{~m} /$ year average rate. In the satellite images these variations were smaller, with $+0.04 \mathrm{~m} /$ year average rate. The adoption of two analyzes in different time scales allowed different evolutions patterns identification for the same shoreline. Modifications were significant in the 72 years period covered by aerial photographs, suggesting an apparent progradation tendency in a decadal scale. In 10 years satellite image series changes were less visible, with irregular variations possibly induced by specific events.
\end{abstract}

Key words: coastal inundation; DSAS; spatial analysis.

\section{Introdução}

As transformações que ocorrem na linha de costa, incluindo suas mudanças de posição, decorrem da ação de variáveis que podem ser tanto naturais quanto antrópicas (Muehe 1995). A compreensão do comportamento dessas variáveis é importante por tratar-se de um ambiente que apresenta considerável pressão de uso e densidade populacional, encontrando-se ainda exposto aos eventos de erosão e inundação que incidem sobre a zona costeira.
Dentre os descritores que podem ser avaliados para identificar áreas suscetíveis a inundações costeiras, destaca-se a análise da taxa de variação da linha de costa através de aerofotografias e imagens de satélite. Vários estudos já foram desenvolvidos tendo por base esse tipo de abordagem (por exemplo: Absalonsen \& Dean 2011, Virdis et al. 2016, Silva et al. 2016, Kermani et al. 2016) sendo, todavia, raras as investigações focadas nos diferentes comportamentos que a linha de 
costa apresenta quando analisada em escalas temporais distintas (Mazzer \& Dillenburg 2009, Ford 2013).

Em particular, setores costeiros submetidos à incidência restrita de ondas em alguns trechos, como as praias de enseada, podem apresentar variabilidade temporal da linha de costa muito diversa ao longo de seu arco praial (Klein \& Menezes 2001). A Baía de Tijucas (figura 1), localizada no Estado de Santa Catarina, é caracterizada principalmente pelo grande aporte e presença de sedimentos finos, com destaque para a presença de lamas, dado seu elevado grau de confinamento.

O Rio Tijucas é o principal contribuinte de sedimentos para as praias locais, tornando-as diferentes dos setores praiais adjacentes (Almeida 2008). Segundo Klein et al. (2016), as praias da Enseada de Tijucas são as únicas dominadas por marés entre as praias voltadas ao mar aberto do Estado de Santa Catarina, podendo ser classificadas como pertencentes ao tipo "praia + planície lamosa". Destaca-se que esta classificação possui base geomorfológica, não tendo sido efetuada a partir de medidas de amplitudes de maré locais, que inexistem para a área. Para as proximidades da Ilha de Santa Catarina as marés de sizígia habitualmente apresentam amplitudes inferiores a 1,2 $\mathrm{m}$ (Bonetti et al. 2018), podendo haver sobrelevação de igual ordem devido à maré meteorológica (Trucollo 1998).

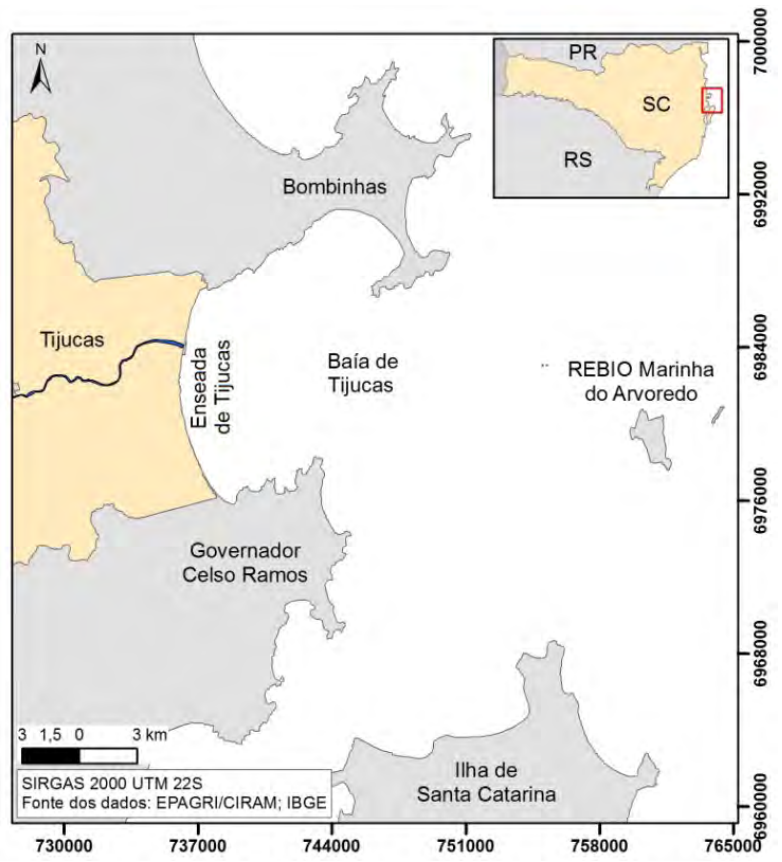

Figura 1. Localização da área de estudo situada no litoral central de Santa Catarina.

A Enseada de Tijucas, situada no interior da baía em questão, pode ser considerada um ambiente costeiro semiabrigado em relação à incidência de ondas. Apenas as ondas oceânicas incidentes do quadrante leste atingem diretamente a costa local, ainda assim sofrendo atenuação causada pelos processos de refração e difração devido à presença das ilhas do arquipélago do Arvoredo. As ondas geradas pelos ventos locais, da mesma forma, provêm somente de nordeste, leste e parcialmente de sudeste, dado o abrigo promovido pelos promontórios rochosos localizados a norte e a sul da área e a presença da Ilha de Santa Catarina. Entretanto, ainda assim a Enseada é suscetível a eventos de inundação costeira associados principalmente à somatória de marés de sizígia, ventos extremos de sul/sudeste e ondas de tempestade (que no conjunto definem a condição extrema de atuação das "marés de tempestades", conforme definidas por Rudorff et al. (2014) para Santa Catarina).

$\mathrm{O}$ entendimento das variáveis naturais e antrópicas que tornam uma área costeira relativamente abrigada, como a Enseada de Tijucas, exposta aos eventos de inundação costeira, contribui para o tema, já que existem poucas pesquisas com esta abordagem em ambientes semiabrigados.

De fato, a área investigada possui registro de ter sofrido, em diversas ocasiões, processo de inundação costeira. Nos últimos 16 anos, ocorreram ao menos nove eventos de transposição da água marinha para a planície à retaguarda, parte dela ocupada por população de baixa renda (Santos 2018). Os efeitos do último evento, ocorrido em 29/10/2016, obteve ampla repercussão local, podendo estes serem visualizados na figura 2.

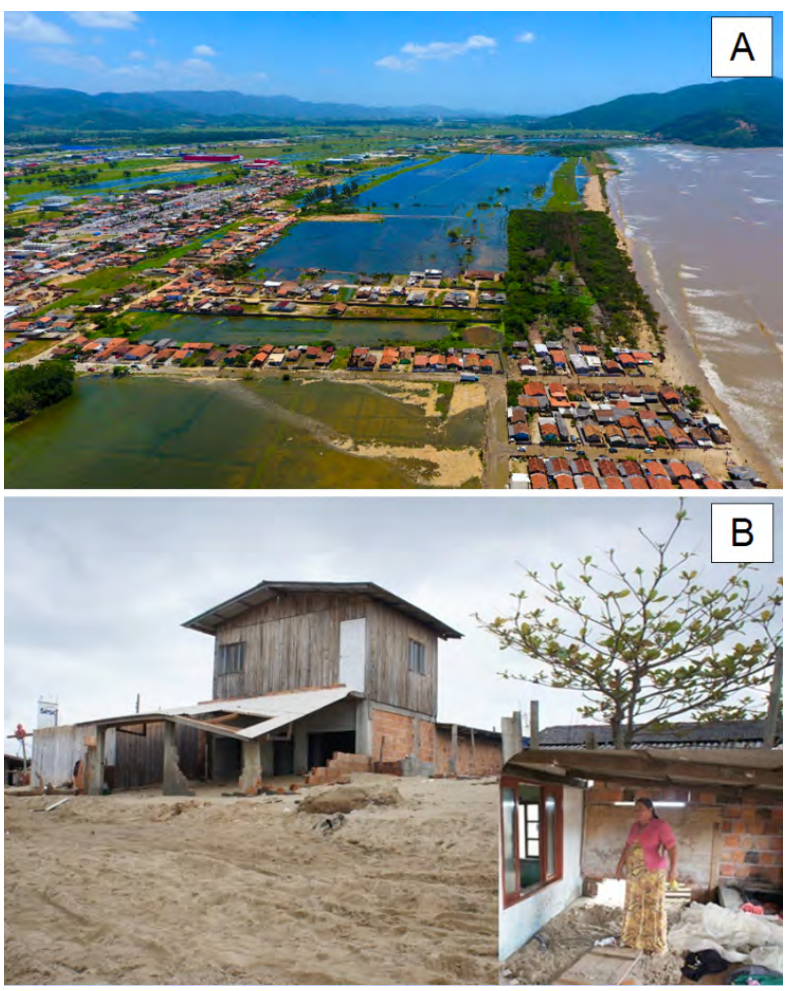

Figura 2. A: Vista para o setor norte da enseada após evento de inundação costeira (Barentin 2016); B: Casa de frente para o mar no setor norte parcialmente soterrada (Souza 2016).

Neste contexto, a pesquisa apresentada tem por objetivo avaliar as variações na posição da linha de costa quando analisadas a partir de duas séries representativas de intervalos temporais distintos, associados às escalas interanual e interdecadal. 


\section{Materiais e métodos}

Para determinar a variação da linha de costa da Enseada de Tijucas utilizou-se a extensão Digital Shoreline Analysis System (DSAS), desenvolvida pelo Serviço Geológico dos Estados Unidos (Thieler et al. 2009) e que opera no sistema ArcGIS(C) da empresa ESRI. Essa extensão possibilita calcular estatisticamente taxas de recuo ou avanço da linha de costa ao longo do tempo.

Optou-se por fazer duas análises da variação da linha de costa local tendo-se em conta os distintos intervalos de tempo e escalas das aerofotografias e imagens de satélite utilizadas. Aerofotografias com espaçamento temporal de 20 a 30 anos foram utilizadas para a "Análise A", sendo estas provenientes da Marinha dos EUA/União (escala 1:30.000), Secretaria de Planejamento e Gestão do Estado (1:25.000), Departamento Nacional de Produção Mineral (1:25.000) e da Secretaria de Desenvolvimento Sustentável (SDS) (1:10.000), referentes aos anos de 1938, 1957, 1978 e 2010, respectivamente. Para a “Análise B" foram utilizadas as imagens de satélite de alta resolução com espaçamento de 1 a 4 anos obtidas através do Google Earth Pro (satélite QuickBird da empresa DigitalGlobe, com resolução espacial multiespectral de $2,4 \mathrm{~m}$ e pancromático de $0,6 \mathrm{~m}$ ) e o aerolevantamento da SDS (2010), que possui resolução semelhante a essas imagens de satélite.

As aerofotografias/imagens de satélite foram georreferenciadas, utilizando-se como base o aerolevantamento da (SDS) de 2010 (ortorretificado). $\mathrm{O}$ erro considerado para o georreferenciamento, de aproximadamente 1 metro, foi baseado em Araujo et al. (2008). Posteriormente, os erros médios (quadro 1) obtidos foram utilizados para o preenchimento do campo das incertezas no DSAS.

Quadro 1. Ano e erro médio do georreferenciamento das aerofotografias* e imagens de satélite utilizadas.

\begin{tabular}{cc}
\hline Ano & Erro médio (m) \\
\hline $1938^{*}$ & 1,11 \\
\hline $1957^{*}$ & 0,9 \\
\hline $1978^{*}$ & 0,84 \\
\hline 2005 & 0,54 \\
\hline 2009 & 0,60 \\
\hline 2011 & 0,52 \\
\hline 2014 & 0,68 \\
\hline 2015 & 0,64 \\
\hline
\end{tabular}

Este campo refere-se às incertezas na posição da linha de costa provenientes das influências naturais (vento, ondas, marés) e de medidas (erros de digitalização e georreferenciamento) (Thieler et al. 2009). Para as aerofotografias de 2010, utilizou-se um valor arbitrário para preenchimento do DSAS, o erro utilizado foi de 0,01m, com base em Muler (2012).

Após o georreferenciamento das aerofotos/imagens, vetorizou-se as linhas de costa dos anos 1938, 1957, 1978 e 2010, de acordo com as definições propostas por Kraus \& Rosati (1997). Utilizou-se como referência a linha da vegetação no pós-praia pois, dada a baixa declividade local, considerou-se que a imprecisão poderia ser maior se adotado o habitual indicador «limite terra/água" (Muehe \& Klumb-Oliveira 2014). Todavia, no setor sul da área de estudo, devido à dificuldade na identificação da vegetação nas aerofotos mais antigas se optou por utilizar o limite terra/água, que ainda assim permitiu boa comparabilidade entre os dados, conforme avaliado visualmente. Para todas as imagens de satélite foi possível utilizar apenas a linha de vegetação para traçar a série de linhas de costa.

A linha de base, de onde partem os transectos (figura 3), foi vetorizada no interior do continente, distante de todas as linhas de costa. Em ambas as análises as linhas de costa e de base possuem uma ruptura devido à presença do Rio Tijucas, localizado entre os transectos 17 e 19.

A partir das intersecções entre os transectos e as linhas de costa vetorizadas é determinada a variação da linha de costa e feito o cálculo da taxa de variação da mesma. Os transectos ao longo da linha de costa, gerados pelo DSAS, foram espaçados de 200 em 200 metros, com comprimento de 450 metros a partir da linha de base.

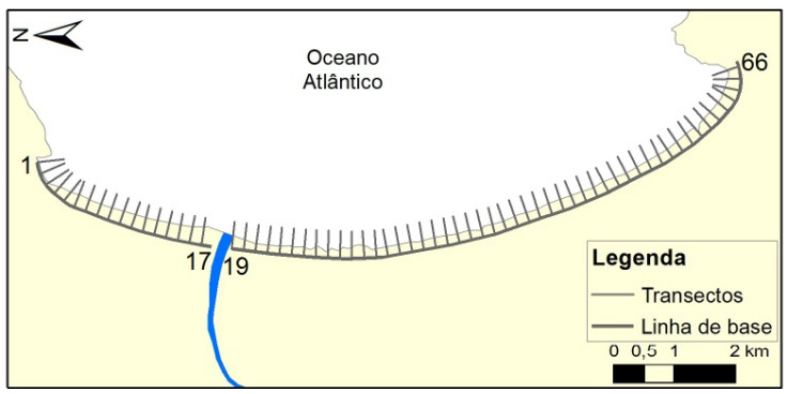

Figura 3. Transectos gerados para os cálculos. Os números correspondem à quantidade de transectos utilizados (66, no total).

Dentre os métodos disponíveis pelo DSAS para o cálculo da taxa de variação da linha de costa, optou-se por o método do weighted linear regression (regressão linear ponderada), que atribui maior peso às linhas de costa que possuem incertezas menores.

Os valores da taxa de variação da linha de costa obtidos para cada transecto, assim como a suscetibilidade da linha de costa em relação a essa variável, foram classificados em três grupos pelo método quebra natural de Jenks (natural breaks), que busca, no processo de agrupamento dos casos, minimizar a variação dentro das classes e maximizar a variação entre as classes (Jenks \& Caspall 1971). Os valores de distância da linha de base às linhas de costa foram exportados para uma planilha eletrônica, para elaboração de gráficos da variação da posição inicial e final entre um ano e outro e para identificar onde houve avanço ou recuo da linha de costa.

\section{Resultados e discussão}

O resultado obtido pelo cálculo da variação em escala decenal (figura 4A) entre a linha de costa mais antiga (1938) e a mais nova (2010) mostra que houve avanço 
na maior parte da área investigada, com aumento em alguns trechos de até 235 metros, principalmente nos setores sul e central, e recuo em uma pequena área do setor norte com valores de até $15 \mathrm{~m}$. Tal fato pode estar relacionado ao clima de ondas anteriormente descrito, que induz um processo de transporte sedimentar de norte para sul. Para o setor norte, considerando como divisor de setores o Rio Tijucas, a largura da linha da costa nas áreas que avançaram não passou de $150 \mathrm{~m}$.

O resultado da análise da variação da linha de costa em escala interanual (figura 4B), de 2005 para 2015, aponta ter havido maior recuo da costa nos setores central e sul (transectos 19 a 66), chegando a 100 metros de diferença. Para as áreas com avanço da linha de costa, a maioria dos valores variou entre 25 e 50 metros.

Para a Análise A (figura 5A), entre os anos de 1938 a 1957 os maiores avanços estão presentes entre os setores central e sul, que incrementaram a costa com valores de 50 a 150 metros. Também estão presentes algumas áreas com recuo da linha de costa, mas que não ultrapassam $50 \mathrm{~m}$ de extensão.

Para os anos de 1957 a 1978 houve avanço na maior parte da linha de costa, destacando novamente os maiores avanços para o setor sul e margem direita do Rio Tijucas, com valores de 100 a cerca de 250 metros.

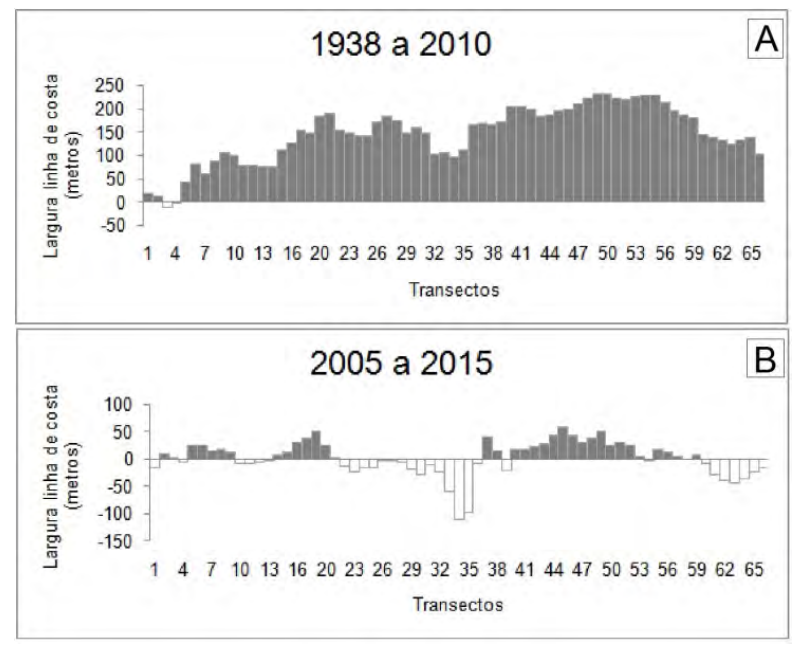

Figura 4. Gráfico com a diferença da linha de costa de 1938 e 2010 (Análise A) e de 2005 e 2015 (Análise B).

Já a diferença entre os anos de 1978 a 2010 aponta a predominância de áreas em que a linha de costa recuou, com os maiores valores de recuo a partir da margem direita do Rio Tijucas em direção ao sul da enseada, com valores que chegam a 115 metros. $\mathrm{O}$ avanço que ocorreu na linha de costa da margem esquerda do rio foi de até 50 metros.

No geral, o acompanhamento das linhas de costas extraídas das aerofotografias mostra que a área de estudo está avançando na escala temporal considerada. Todavia, quando se analisa a diferença entre cada conjunto de aerofotos é possível observar momentos de avanço e de recuo ao longo do tempo.

Para a Análise B (figura 5B), as modificações ocorridas são mais discretas, sem que fique evidenciada uma clara tendência de recuo ou avanço, quando se comparada à Análise A.

Nos anos de 2005 a 2009 os maiores valores obtidos de avanço da linha de costa foram entre o setor central e sul, com 30 a 70 metros no geral, e o recuo da costa atingiu até 20 metros.

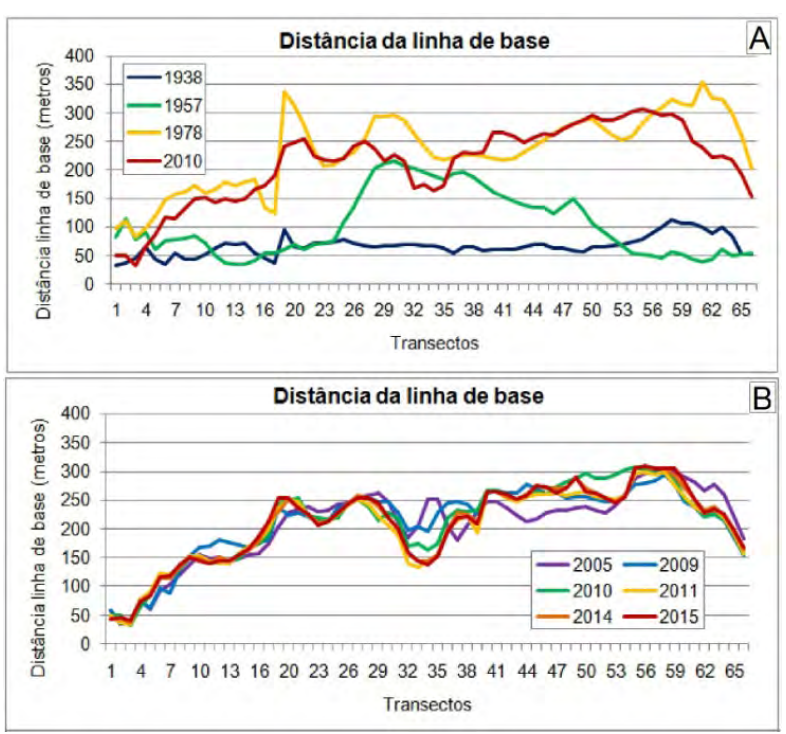

Figura 5. Distância entre as linhas de costa da linha de base (considerada eixo zero) referentes à Análise A e Análise B.

Para os anos de 2009 a 2010 e de 2010 a 2011 o recuo da linha de costa foi maior que o avanço, com valores em torno de 20 a 60 metros de recuo da costa para os anos 2009/2010 e de 10 a 50 metros para 2010/2011.

De 2011 a 2014 os avanços estiveram mais presentes no setor central e sul, com valores de 15 a 30 metros, e no setor norte o recuo foi de até 10 metros.

Nos anos de 2014 a 2015 o maior avanço ocorreu na margem direita do Rio Tijucas, próximo ao transecto 19 e, no geral, o restante da linha de costa sofreu recuo em torno de 10 metros.

Quanto à taxa de variação média para toda a linha de costa, a Análise A apresenta taxa média de $+1,62 \mathrm{~m} /$ ano e a Análise B, $+0,04 \mathrm{~m} /$ ano. Já para cada transecto, com base na classificação da taxa de variação da linha de costa pelo método da quebra natural de Jenks (natural breaks), a análise proveniente das aerofotos interdecadais (figura 6A) apresenta menores taxas de recuo quando comparada com as de imagens de satélite - interanuais (figura 6B).

A partir dos resultados obtidos, pode-se propor uma classificação da linha de costa local em três grupos que apresentam taxas distintas de variação: alta, moderada ou baixa, representados pelas cores vermelha, amarela e verde, respectivamente, na figura 6. Para a análise A, as taxas de $-0,7$ a $+0,8$ metros/ano foram consideradas de classe "alta", de $+0,9$ a $+2,1$ metros/ano "média" e de $+2,2$ a $+3,5$ metros/ano "baixa". Já para a análise B, -11,6 a -7,6 metros/ano classe "alta", de -7,5 a $+0,6$ metros/ano "média" e de $+0,7$ a $+5,4$ metros/ano "baixa". 


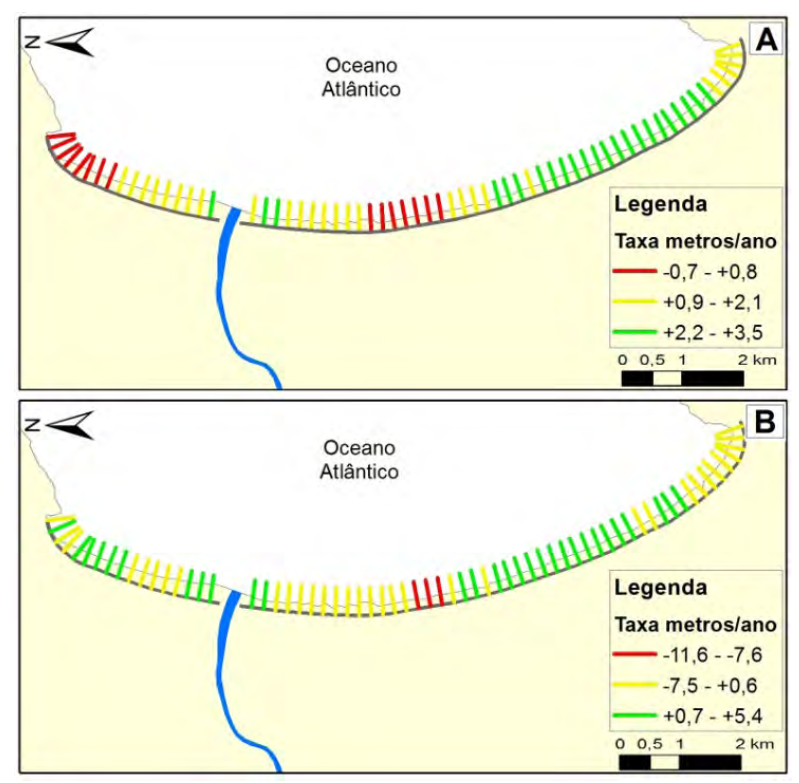

Figura 6. Taxa de variação da linha de costa. A: 1938 a 2010; B: 2005 a 2015 . As cores dos transectos em vermelho, amarelo e verde indicam, respectivamente, suscetibilidade alta, moderada e baixa.

Pode-se argumentar que, para a determinação da suscetibilidade, a escala temporal que melhor se adequaria para a área de estudo seria a análise B. Isso decorre da quantidade de imagens disponíveis, por ela estar mais próxima temporalmente da realidade atual e por representar o cenário mais pessimista em relação à alta taxa de recuo da linha de costa, ainda que a análise das aerofotos apresente mais transectos como de suscetibilidade alta.

No âmbito desta pesquisa, suscetibilidade é compreendida como a predisposição natural de um sistema ser afetado por um determinado fator de ameaça, primariamente em decorrência das condições físicas locais (UNDP 2004). Neste caso, considera-se como efeito do perigo costeiro a transposição do póspraia por ondas em períodos de sobrelevação do nível do mar e consequente inundação à retaguarda.

Esse mesmo processo foi investigado por Lins de Barros \& Muehe (2010) na Região dos Lagos - Rio de Janeiro, tendo os autores utilizado como indicadores de susceptibilidade a exposição à transposição do cordão litorâneo por ondas, evidências de erosão costeira e registros recentes de inundações e alagamentos.

Embora a taxa de variação da linha de costa seja apenas um dos indicadores da susceptibilidade local, possuindo relação com o descritor "evidências de erosão costeira" proposto por Lins de Barros \& Muehe (2010), o mesmo pode ser considerado uma boa alternativa de fator primário de exposição a ser investigado. $\mathrm{Na}$ área, sobretudo devido à extensa planície que ocorre à retaguarda e sobre a qual ocorrem diversos tipos de uso, o progressivo recuo da linha de costa estabelece a condição física para que a transposição por ondas venha a impactar progressivamente as atividades humanas (ligadas à agricultura e urbanização).
Reconhece-se, todavia, que a efetiva suscetibilidade local somente poderá ser plenamente estabelecida após a incorporação de variáveis complementares, como a análise da exposição à ação de ondas, a determinação da variabilidade altimétrica local e caracterização do pós-praia.

Quanto à análise A, esta é importante para o entendimento das alterações recentes da posição da linha de costa na área de estudo, principalmente no setor urbano (figura 7), destacando-se parte das estruturas e residências situadas atualmente em um trecho da linha de costa que somente a partir de 1978 obteve configuração semelhante à atual.

As distintas análises aplicadas nesta pesquisa permitiram visualizar evoluções diferenciadas para a mesma área de estudo, assim como Mazzer \& Dillenburg (2009) ao analisarem cinco praias da costa sudeste da Ilha de Santa Catarina nas escalas interdecadal e interanual, também notaram que a utilização de diferentes intervalos temporais pode levar a resultados distintos. Percepção semelhante resultou a pesquisa de Esteves et al. (2006), que analisaram a variação da linha de costa do Rio Grande do Sul nas escalas sazonal e interanual. Os autores supracitados identificaram, em seu trabalho, que a energia das ondas e a frequência de tempestades influenciaram as variações sazonais da linha de costa, enquanto que eventos de El Niño-Oscilação Sul estavam associados às alterações ocorridas na escala interanual. Crowell et al. (1993) analisaram duas áreas da costa de Maryland, Estados Unidos, e concluíram que as taxas de erosão para análises feitas a longo prazo são mais suaves do que as de curto prazo, assim como encontrado para a linha de costa da Enseada de Tijucas.

Apesar de tratar-se de um ambiente distinto, resultado semelhante foi encontrado por Ford (2013) que avaliou o Atol Wojte, situado nas Ilhas Marshall, utilizando igualmente escalas temporais distintas na análise. Naquela pesquisa, no período de 67 anos analisados (1945-2012), a acreção da linha de costa prevaleceu sobre o recuo da mesma, enquanto que para a análise efetuada entre 2004 e 2012, grande parte da linha de costa estava em processo de recuo.

Processos interdecadais, associados à escala de investigação de uma das séries temporais aqui investigadas, podem refletir posições da linha de costa decorrentes de algumas das forçantes anteriormente mencionadas, assim como tendências de longo prazo resultantes de mudanças no aporte de sedimentos a partir da bacia hidrográfica ou ainda de padrões de ocupação costeira que poderiam ter alterado o balanço sedimentar local. Desta forma, seu uso em análises de vulnerabilidade deve ser efetuado com ressalvas, pois séries com aerofotos largamente espaçadas podem estar representando posições de linha de costa decorrentes tanto de tendências de longo prazo quando de variações de alta frequência, dependendo do momento da aquisição. 

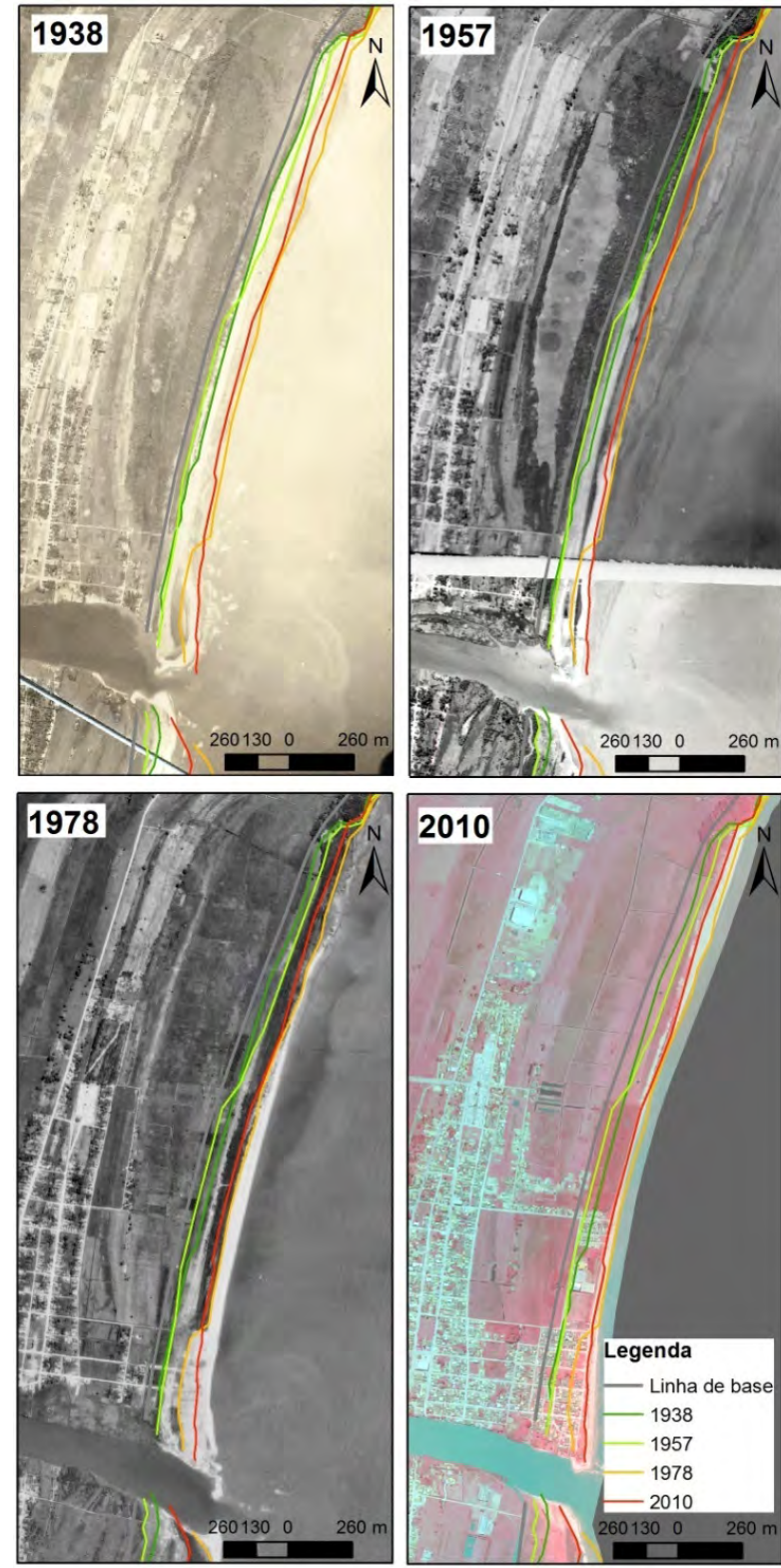

Figura 7. Evolução da mancha urbana e da linha de costa proveniente da Análise A.

\section{Conclusões}

Embora a Enseada de Tijucas possa ser considerada um ambiente costeiro semiabrigado, a mesma sofre, com alguma regularidade a ação de eventos de inundação costeira associados a marés de tempestade.

Dentre os descritores utilizados na identificação de áreas suscetíveis a este processo, destaca-se a análise da taxa de variação da posição da linha de costa.

A investigação realizada neste trabalho focou nos diferentes comportamentos da linha de costa quando analisada a partir de dados representativos de escalas temporais distintas. A extensão DSAS possibilitou esta análise e se mostrou uma rápida e eficiente opção metodológica. As distintas escalas de investigação permitiram visualizar processos morfológicos efetivamente diferenciados atuando na área de estudo.

Os maiores avanços da linha de costa na Enseada de Tijucas ocorreram na escala interdecadal (análise A), com valores de avanço de ordem superior a $200 \mathrm{~m}$, quando se compara a diferença entre a linha de costa mais antiga (1938) e a mais nova (2010). Para a escala interanual (análise B), as mudanças foram mais sutis nos 10 anos analisados.

A maior taxa de avanço da linha de costa na área de estudo ocorreu na análise de 72 anos, que apresentou valor médio de $+1,62 \mathrm{~m} /$ ano. Para o intervalo de 10 anos a taxa média foi de $+0,04 \mathrm{~m} /$ ano.

Com base nos resultados obtidos, sugere-se levar em consideração, para análise da suscetibilidade da área investigada visando o planejamento costeiro, a análise de 10 anos baseada em imagens de satélite recentes e com maior frequência de imageamento, pois essas parecem estar indicando tendências mais atuais dos processos locais. Entretanto, a análise A é importante para compreensão da evolução local, devendo ser considerada em planos de gestão de longo prazo.

\section{Referências}

Absalonsen L., Dean R. G. 2011. Characteristics of the shoreline change along Florida sandy beaches with an example for Palm Beach County. Journal of Coastal Research, 27(6A), 16-26.

Almeida D. C. 2008. Diagnóstico da distribuição de sedimentos finos e processos físicos associados na Baía de Tijucas. Dissertação de Mestrado. Pós-Graduação em Ciência e Tecnologia Ambiental, Centro de Ciências Tecnológicas, da Terra e do Mar, Universidade do Vale do Itajaí, 85p.

Araujo R. S., Silva G. V., Freitas D., Klein A. H. F. 2008 Georreferenciamento de fotografias aéreas e análise da variação de linha de costa. In: Alcántara-Carrio J., Correa I. D., Isla F., Alvarado M., Klein A. H. F., Cabrera J. A. (eds) Metodologias en Teledetección Aplicada a la Prevención de Amenazas Naturales en el Litoral, Valencia, 237-253p.

Barentin L. 2016. Risco de ressaca persiste neste domingo. Disponível em: http://jornalrazao.com/materia/risco-de-ressaca-persisteneste-domingo-1435. Acessado em novembro 2016.

Bonetti J., Rudorff F. M., Campos A. V., Serafim M. B. 2018 Geoindicator-based assessment of Santa Catarina (Brazil) sandy beaches susceptibility to erosion. SI: Management Strategies for Coastal Erosion Problems. Ocean \& Coastal Management, 156:198-208.

Crowell M., Leatherman S. P., Buckley M. K. 1993. Shoreline change rate analysis: long term versus short term data. Shore and Beach, 61(2):13-20.

Esteves L. S., Williams J. J., Dillenburg S. R. 2006. Seasonal and interannual influences on the patterns of shoreline changes in Rio Grande do Sul, Southern Brazil. Journal of Coastal Research, 22(5), 1076-1093.

Ford M. 2013. Shoreline changes interpreted from multi-temporal aerial photographs and high resolution satellite images: Wotje Atoll, Marshall Islands. Remote Sensingof Environment, 135:130-140.

Jenks G. F., Caspall F. C. 1971. Error on choroplethic maps: definition, measurement, reduction. Annals of the Association of American Geographers, 61(2):217-244.

Kermani S., Boutiba M., Guendouz M., Guettouche M. S, Khelfani D. 2016. Detection and analysis of shoreline changes using geospatial tools andautomatic computation: Case of Jijelian sandy coast (East Algeria). Ocean \& Coastal Management, 132:46-58.

Klein A. H. F., Menezes J. T. 2001. Beach morphodynamics and profile sequencefor a Headland Bay coast. Journal of Coastal Research, 17(4), 812-835. 
Klein A. H. F., Short A. D., Bonetti J. 2016. Santa Catarina beach systems. In: Short A. D., Klein A. H. F. (eds) Brazilian Beach Systems. Chapter 17. Coastal Research Library 17. Springer, Switzerland, 465-506p.

Kraus N., Rosati J. 1997. Interpretation of shoreline-position data for coast al engineering analysis. Coastal Engineering Technical Note. CETN II-39, U.S. Army Engineer Waterways Experimental Station, 13p.

Lins de Barros F. L., Muehe D. 2010. Avaliação local da vulnerabilidade e riscos de inundação na zona costeira da Região dos Lagos, Rio de Janeiro. Quaternary and Environmental Geosciences, 02(1):55-66.

Mazzer A. M., Dillenburg S. 2009. Variações temporais dalinha de costa em praiasarenosas dominadas por ondas do sudeste da Ilha de Santa Catarina (Florianópolis, SC, Brasil). Pesquisas em Geociências, 36(1):117-135.

Muehe D. 1995. Geomorfologia Costeira. In: Guerra A. J. T., Cunha S. B. da (eds) Geomorfologia: uma atualização de bases e conceitos. Bertrand Brasil, Rio de Janeiro, 253-303p.

Muehe D., Klumb-Oliveira L. 2014. Deslocamento da linha de costa versus mobilidade praial. Quaternary and Environmental Geosciences, 5(2):121-124.

Muler M. 2012. Avaliação da vulnerabilidade de praias da Ilha de Santa Catarina a perigos costeiros através da aplicação de um índice multicritério. Dissertação de Mestrado. Pós-Graduação em Geografia, Departamento de Geociências, Universidade Federal de Santa Catarina, 213p.

Rudorff F. M., Bonetti J., Moreno D. A., Oliveira C. A. F, Murara P. G. 2014. Maré De Tempestade. In: Herrmann M. L. P. Atlas de desastres naturais do Estado de Santa Catarina: período de 1980 a 2010. IHGSC/Cadernos Geográficos, Florianópolis, 151-154p.
Santos E. C. 2018. Análise da suscetibilidade e da vulnerabilidade costeira de um sistema semiabrigado a eventos extremos: Enseada de Tijucas - Santa Catarina. Dissertação de Mestrado. Pós-Graduação em Geografia, Departamento de Geociências, Universidade Federal de Santa Catarina, 100p.

Silva G. V., Muler, M., Prado, M. F. V., Short, A., Klein, A. H. F., Toldo Jr., E. 2016. Shoreline change analysis and insight into the sediment transport path along Santa Catarina Island north shore, Brazil. Journal of Coastal Research, 32(4):863-874

Souza C. E. 2016. Praia invade casas no bairro da Praça. Disponível em: http://www.daquitijucas.com.br/noticias/613-praia-invadecasas-no-bairro-da-praca. Acessado em novembro 2016.

Thieler E. R., Himmelstoss E. A., Zichichi J. L., Ergul A. 2009. Digital Shoreline Analysis System (DSAS) version 4.0 - An ArcGIS extension for calculating shoreline change: U.S. Geological Survey Open-File Report, 2008-1278.

Trucollo E. C. 1998. Maré meteorológica e forçantes atmosféricas locais em São Francisco do Sul - SC. Dissertação de Mestrado. Pós-Graduação em Engenharia Ambiental, Departamento de Engenharia Sanitária e Ambiental, Universidade Federal de Santa Catarina, 100p.

UNDP - United Nations Development Programme. 2004. Reducing Disaster Risk: A Challenge for Development. John S. Swift, New York.

Virdis S. G. P., Oggiano G., Disperati, L. 2012. A geomatics approach to multitemporal shoreline analysisin Western Mediterranean: the case of Platamona-Maritza Beach (Northwest Sardinia, Italy). Journal of Coastal Research, 28(3):624-640. 